\title{
Stark shift of the Cs clock transition frequency: A new experimental approach
}

\author{
Jean-Luc Robyr, Paul Knowles, and Antoine Weis \\ Fribourg Group of Atomic Physics \\ Department of Physics \\ University of Fribourg, CH-1700 Fribourg \\ Email: jean-luc.robyr@unifr.ch
}

\begin{abstract}
The blackbody radiation shift, a manifestation of the Stark effect due to blackbody radiation, contributes to the systematic uncertainty in Cs-based frequency standards at a level of $1 \times 10^{-15}$. Few measurements of the third order scalar electric polarizability of the Cs ground states, mainly responsible of the AC Stark shift in atomic clocks, have given better than $10 \%$ accuracy. We report progress in the development of a fully optical Ramsey pump-probe measurement in a thermal atomic beam, based on coherent population trapping (CPT), for the measurement of the third order scalar and tensor polarizabilities of the Cs ground states. We give details of the apparatus and measurement techniques as well as our first $500 \mathrm{~Hz}$ half-period Ramsey fringes.
\end{abstract}

\section{INTRODUCTION}

The frequency of the ground state hyperfine splitting in Cs has defined the second since 1967 (ISO 31-1), and the understanding and control of the systematic effects [1], [2] which perturb that frequency is of the highest importance for time and frequency metrology. The Stark shift, a change of atomic level energies due to an applied electric field, has been considered and controlled in atomic clocks since the early ' 80 s when it was realized that the AC Stark shift arising from the blackbody radiation spectrum of the finite temperature surroundings of the clock would shift the resonance [3]. The recent AC and DC Stark shift measurements have shown a discrepancy in the measured value for the third order scalar polarizability $\alpha_{0}^{(3)}(F)$ [4]-[7] and calls for clarification of the issue have appeared [1]. The commercial availability of optical phase modulation devices make straightforward the replacement of the microwave cavity used in atomic frequency standards (either beam or fountain clocks), by a bichromatic, phase coherent, light source, where the frequency difference between the two light field is equal to the microwave frequency.

We are presently optimizing a CPT-based Ramsey pumpprobe experiment on an atomic $\mathrm{Cs}$ beam similar to that introduced in 1982 by Thomas et al. [8] for a $\mathrm{Na}$ atomic beam, and used recently by Fuchs et al. [9] with Li atoms. The technique will be used to measure $\alpha_{0}^{(3)}$ of the Cs atom that is responsible for the blackbody radiation shift in Csbased frequency standards. We are confident in our ability to achieve a measurement below $1 \%$ since a similar version of the method was successfully used by us to measure the thirdorder tensor polarizability, $\alpha_{2}^{(3)}$, in Cs, a value roughly two orders of magnitude smaller than $\alpha_{0}^{(3)}$ [10], [11].
The basic principle can be viewed as the measurement of the relative phase difference which accumulates over time resulting from a tiny frequency difference between two oscillators. The first frequency is the intrinsic frequency of the atom itself, $\nu_{1}$, while the second, $\nu_{2}$, is generated by a microwave synthesizer. A first interaction between the microwave and the atom sets the phase difference to zero. Then, the atom enters a free precession zone for a time $T$ where it accumulates a phase difference $\Phi=2 \pi\left(\nu_{1}-\nu_{2}\right) T$ with respect to the microwave. The accumulated phase is finally probed by the same microwave frequency. Every change in the energy structure of the atom due to an external perturbation during the free evolution, e.g., by an electric field, will result in a phase difference at the probe region and will be detected.

In our case, the atomic reference will be the hyperfine coherence oscillation, prepared by a CPT interaction, and the microwave signal will come from a generator referenced to an atomic clock. A static electric field will be applied in the free evolution zone and the resulting phase shift, due to the Stark effect, will be probed with the same bichromatic field. Due to the thermal distribution of the atomic velocities in an atomic beam, the free evolution time, and thus the final accumulated phase, has to be averaged over the velocity distribution of the atoms of the beam. Finally, the Stark phase shift will be given by

$$
\Delta \Phi=\frac{\Delta E}{\hbar} L\left\langle\frac{1}{v}\right\rangle
$$

for $\Delta E$ the energy difference induced by the Stark effect, $L$ the free atoms evolution distance, and $\left\langle\frac{1}{v}\right\rangle$ the average of the inverse atomic velocity.

The signal of interest that will let us measure the effect is the transmission or florescence intensity of the laser at the second interaction, since the phase difference between the atomic coherence and the microwave produces a variation of the optical absorption coefficient. We record the transmitted light intensity versus the microwave frequency. When the microwave frequency is scanned around the clock frequency, the absorption of the atomic beam shows a Ramsey-type interference pattern [12]. Any additional phase difference produces specific fringe shifts that will be measured. It is important to note that we are not interested in the absolute value of the resonance frequency as in the case of atomic 
clocks, but only in the differential shift induced by the electric field, which will greatly simplify our task.

The energy shift produced by a DC electric field $\mathbb{E}$ is described using the electric polarizability $\alpha$ as $\Delta E\left(n, L_{J}, F\right)=$ $-\frac{1}{2} \alpha \mathbb{E}^{2}$. The constant $\alpha$ is traditionally expanded in power series, both in multipole order (subscript) and perturbation order (superscript). Neglecting the (small) quadrupole hyperfine interaction, the lowest order terms (i.e., third) which affect the Cs hyperfine ground state transitions, with $\Delta m=0$, produce a frequency shift [13], [14]

$\delta \nu=-\frac{\mathbb{E}^{2}}{2 h}\left[\frac{16}{7} \alpha_{0}^{(3)}(F=4)+\alpha_{2}^{(3)}(F=4) \frac{3 m^{2}-16}{28} f(\theta)\right]$,

where $\alpha_{0}^{(3)}$ and $\alpha_{2}^{(3)}$ are the third order scalar and tensor polarizabilities, $m$ is the magnetic quantum number defined by the quantization axis (magnetic field), and $f(\theta)=3 \cos ^{2}(\theta)-1$ is a function of the relative angle between $\mathbb{E}$ and the magnetic field. Note that $f(\theta)$ averages to zero for isotropic blackbody radiation. For an electric field of $20 \mathrm{kV} / \mathrm{cm}$, the expected scalar shift in Cs is $830 \mathrm{~Hz}$, and the tensor shift about $3 \mathrm{~Hz}$.

\section{EXPERIMENTAL SETUP}

\section{A. Vacuum system and cesium beam}

The total length of the vacuum tube represented in Fig. 1 is $1.2 \mathrm{~m}$. An internal pressure of $5 \times 10^{-8}$ mbar is held by two turbo pumps with $80 \mathrm{l} / \mathrm{s}$ and $70 \mathrm{l} / \mathrm{s}$ pumping speeds. The beam is produced by a cesium reflux oven [15] heated to $170{ }^{\circ} \mathrm{C}$. The atomic vapor escapes the oven through a $70 \mathrm{~mm}$ long collimator containing 5 discrete $2 \mathrm{~mm}$ diameter apertures. The oven is mounted on a three-point kinetic mount to allow angular alignment of the beam.

The first vacuum region after the oven collimator is used for preparation of the beam. Large windows give optical access to the beam in both vertical and horizontal directions perpendicular to the beam propagation. They will be used for optical preparation of the atomic state or/and transverse atomic velocity laser cooling. Two additional slit collimators are used to give the beam a rectangular profile. Their widths and heights can be modified to adapt the beam geometry to our needs.

The preparation zone is followed by the magnetically shielded Ramsey arrangement with preparation and interrogation zones. A pair of $10 \mathrm{~mm}$ diameter windows are used for the pump and probe beams. The separation between the pump and probe region is $300 \mathrm{~mm}$. The last section of the vacuum system consists of the high voltage feedthrough and is reserved for optional beam diagnosis, e.g., probing the beam spatial distribution and density with a Langmuir-Taylor hot wire detector.

\section{B. Lasers}

Two lasers are used at the moment, a Cs $D_{1}$ extended cavity diode laser for the Ramsey pump-probe experiment and a Cs $D_{2}$ extended cavity diode laser for hyperfine repumping. Both lasers are stabilized to specific Cs hyperfine transitions using saturated absorption spectroscopy in a cesium vapor cells.

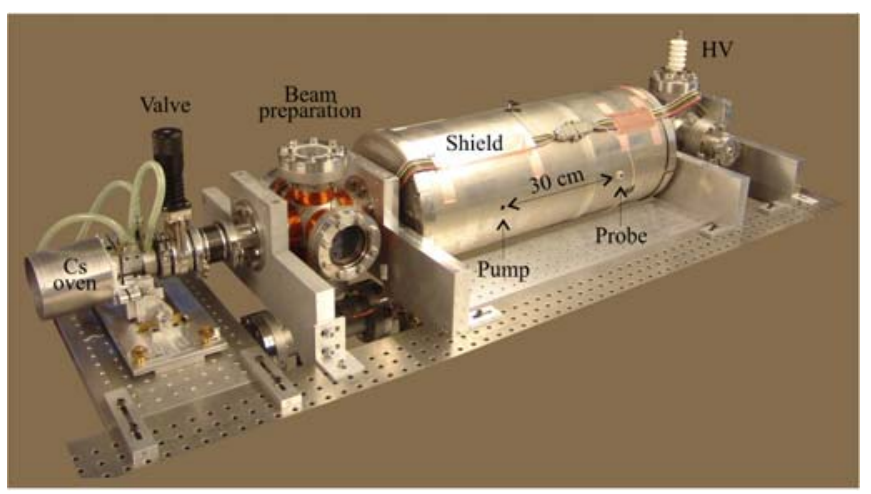

Fig. 1. The atomic beam apparatus with preparation and detection zones At left, the Cs reflux oven in its precision alignment mount. The cross with windows and magnetic field coils is used for the hyperfine state preparation The two-layer magnetic shield is installed with a set of demagnetization coils and has two holes that allow the pump and the probe laser beams to reach the atoms. The high voltage feedthrough is visible at the far right.

\section{Preparation of the atomic beam}

A cesium $D_{2}$ extended cavity diode laser, working at $852 \mathrm{~nm}$ is used for the atomic state preparation via optical pumping. Its purpose is to maximize the population of the two Zeeman states that are involved in the CPT interaction. For improvement of the clock transition signal, a convenient optical pumping transition is the $F=4 \rightarrow F=5$, since it tends to bring the atoms to the $m=0$ level in the $F=4$ state, due to branching ratios that favor decays to lower magnetic quantum numbers. Moreover, this transition is cycling and therefore allows more efficient pumping. The preparation laser wavelength is locked to the atomic transition using saturated absorption in a Cs vapor cell, and about $3 \mathrm{~mW}$ of $\pi$-polarized light power is used for the interaction. In our existing measurements of the preparation effect, the presence in the pump region of a high magnetic field used for the detection of the Ramsey signal caused the pumping efficiency to be suboptimal since the laser linewidth $(<1 \mathrm{MHz})$ was too small to reach all Zeeman transitions, which were separated by $\sim 6 \mathrm{MHz}$. Nevertheless we measured an increase of the Ramsey signal intensity by $20 \%$. Work on improving this is underway.

\section{Coherent population trapping (CPT)}

The phase coherent bichromatic field used to produced the CPT signal is obtained by sending the Cs $D_{1}$ laser beam into a commercial lithium niobate electro-optic modulator (EOM). The modulation source is a microwave synthesizer whose frequency can be scanned around the cesium clock transition frequency. The synthesizer signal is amplified and sent to the EOM crystal. The EOM optical output spectrum contains sidebands that are separated from the carrier by the modulation frequency, and whose amplitudes depend on the applied modulation power. The phase coherence of the carrier and the sidebands is guaranteed by the use of the single input light source. A CPT interaction is obtained when the frequency of the $D_{1}$ laser (the carrier) is locked to a Cs atomic transition 
coupling one of the hyperfine ground states with one of the $6 P_{1 / 2}$ excited states, and the EOM modulation frequency is set to match the transition frequency between the two ground states levels. A sweep of the microwave frequency is used to scan the CPT resonance. It is important to note that the intensity ratio between the two light fields has to be such that the Rabi frequencies of the two transitions are equal. If not, the CPT resonance absorption becomes asymmetric and systematic effects arise [16].

\section{E. The pump-probe experiment}

The mean atomic velocity is given by the oven temperature and is $260 \mathrm{~m} / \mathrm{s}$, giving an average free precession time of $1.2 \mathrm{~ms}$ over $300 \mathrm{~mm}$. A two-layer $\mu$-metal magnetic shield excluded the earth field from the critical experimental volume, leaving a residual magnetic field of about $20 \mathrm{nT}$ in the entire Ramsey region. Two pairs of rectangular Helmholtz coils and a solenoid built around the vacuum tube were used to control the magnetic field. Optical access to the atomic beam for the pump and probe region allowed $7 \mathrm{~mm}$ diameter laser beams to interact with the atoms. The pump and probe laser beams were circularly polarized and the magnetic field was chosen parallel to the light propagation, a configuration chosen to form CPT coupling between states with $\Delta m=0$ without being limited by the two forbidden Zeeman transitions $m=0 \rightarrow m^{\prime}=0$ with $F=F^{\prime}$. The transmission of the probe beam was detected by a photodiode. To diminish the noise in the detection phase sensitive detection was used. The microwave frequency sent into the EOM was frequency modulated at $1 \mathrm{kHz}$ with an appropriate amplitude and the photodiode signal was demodulated by use of a lock-in amplifier. The optical path length of the probe beam, which controls the microwave phase for the probe interaction, could be varied by an optical delay line using a prism mounted on a translation stage.

\section{EXPERIMENTAL RESULTS}

\section{A. CPT pump-probe signal}

Figure 2 shows a typical Ramsey fringe of the clock transition. As expected from the $1.2 \mathrm{~ms}$ free precession time, the half-period of the central fringe is about $500 \mathrm{~Hz}$. The signal was obtained with a $15 \mu \mathrm{T}$ magnetic field parallel to the light path (no electric field was applied). The laser wavelength was locked to the $F=4 \rightarrow F^{\prime}=4$ transition of the Cs $D_{1}$ line. The pump and probe beams were $\sigma^{-}$ polarized with powers of $335 \mu \mathrm{W}$ and $25 \mu \mathrm{W}$, respectively. The present frequency resolution $(\sim 20 \mathrm{~Hz})$ is limited by acoustic noise transmitted by the optics and will be improved following Zeeman state preparation with optical pumping. By increasing the microwave frequency scan, it becomes possible to observe the span of the seven possible CPT transitions between the two Cs ground states. Figure 3 clearly shows six of the seven CPT Ramsey resonances induced by $\sigma^{-}$polarized light. The amplitude distribution asymmetry is due to the $\sigma^{-}$ optical pumping induced by the pump beam which tends to populate states with negative magnetic quantum numbers. The

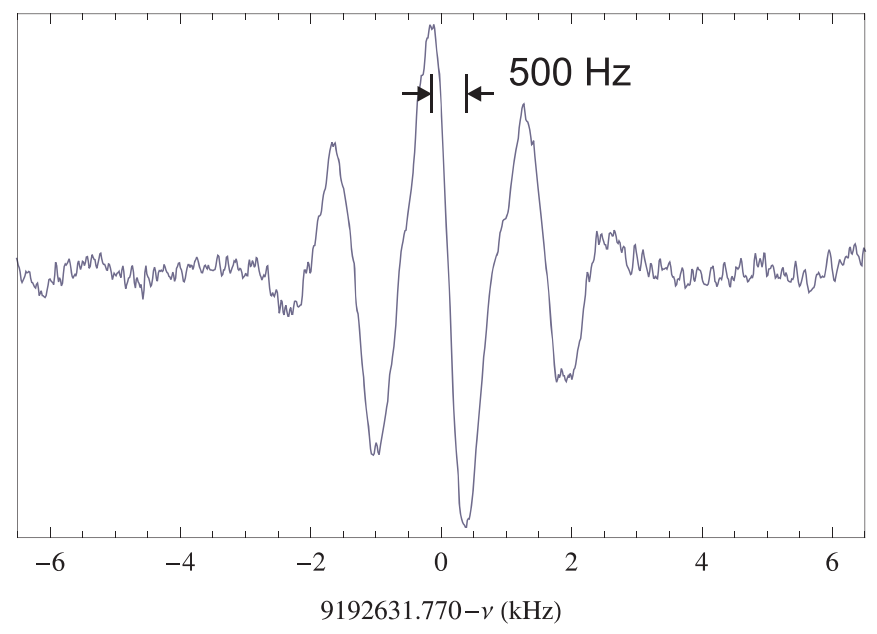

Fig. 2. Ramsey fringes pattern of the cesium clock transition, with a $300 \mathrm{~mm}$ separation between the pump and probe interactions. As expected from the free precession time the central fringe half-period is about $500 \mathrm{~Hz}$. The dispersive lineshape is due to the phase-sensitive detection.

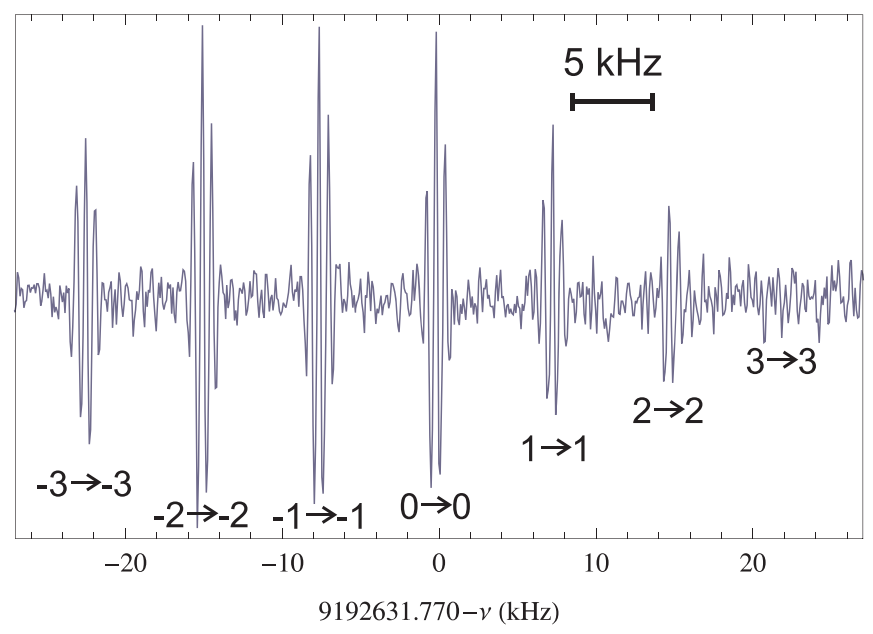

Fig. 3. Six of the seven Ramsey fringes patterns produced by FM-CPT with $\sigma^{-}$pump and probe polarization. The amplitude distribution asymmetry is due to the $\sigma^{-}$optical pumping induced by the pump which tends to populate states with lower magnetic quantum numbers. The depopulated $m_{3}=3 \rightarrow m_{4}=3$ transition is hidden in the signal noise.

$m_{3}=3 \rightarrow m_{4}=3$ Ramsey interference is buried in the noise background.

\section{B. Pump-probe microwave relative phase}

The microwave phase difference between the pump and probe regions has an important effect on the Ramsey signal. It induces a phase shift of the fringes with respect to the signal envelope and makes the signal asymmetric. In this experiment, the microwave is carried by the bichromatic laser light and thus the microwave phase difference depends on the optical path length difference. The wavelength of the microwave is $3.261 \mathrm{~cm}$. By use of a prism mounted on a translation stage, the microwave phase of the probe beam was varied and the effect on the Ramsey pattern recorded. Figure 4 


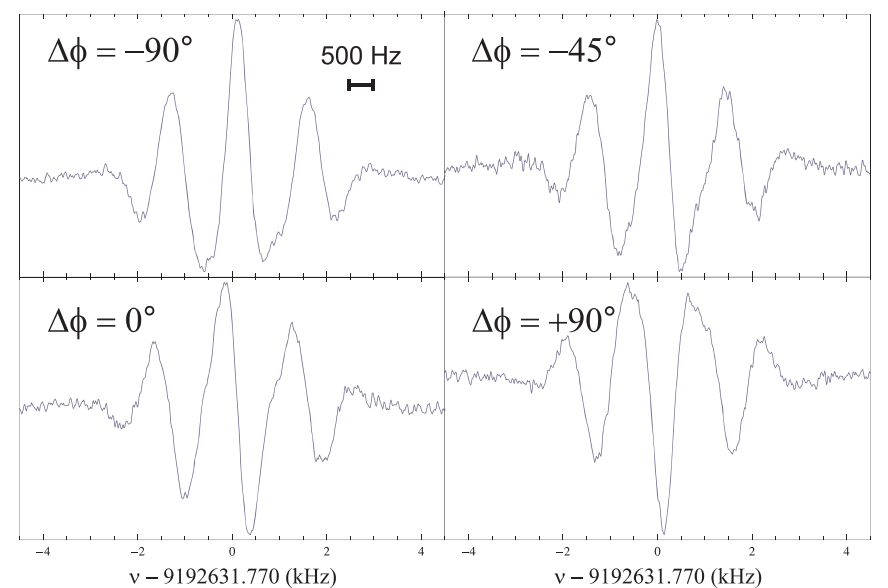

Fig. 4. Data showing the effect of a microwave phase difference $\Delta \phi$ between the pump and probe region. The phase of the microwave at the probe region is changed by varying the optical path length. A variation of optical length of $3.261 \mathrm{~cm}$ corresponds to a phase shift of $2 \pi$. Here are recorded data for $\Delta \phi=-\frac{\pi}{2},-\frac{\pi}{4}, 0$ and $+\frac{\pi}{2}$.

shows the clock transition Ramsey fringes for different relative microwave phase settings.

\section{CONCLUSION}

The results that we have shown so far represent the first step towards the measurement of the Stark effect. The next challenges are the improvement of the signal-to-noise ratio and the installation, calibration, and computer modeling of the $\mathbb{E}$-field. Our goal is to increase the frequency resolution by two orders of magnitude, i.e., from $20 \mathrm{~Hz}$ to $0.1 \mathrm{~Hz}$, which will reach the resolution of our microwave synthesizer. This will be achieved by filtering or eliminating acoustic noise transmitted by the optics, optimizing the phase sensitive detection, improving the signal amplitude by use of optical pumping, and studying the stability over long time averaging.

\section{ACKNOWLEDGMENT}

The authors thank the mechanical and electronics workshops of the Department of Physics of the University of Fribourg for our fruitful collaboration and the Swiss National Science Foundation (SNF \#200021 - 117841) for supporting this project.

\section{REFERENCES}

[1] R. Wynands and S. Weyers, "Atomic fountain clocks," Metrologia, vol. 42, pp. S64-S79, 2005. [Online]. Available: http://www.iop.org/EJ/ abstract/0026-1394/42/3/S08

[2] J. H. Shirley, W. D. Lee, and R. E. Drullinger, "Accuracy evaluation of the primary frequency standard NIST-7," Metrologia, vol. 38 , no. 5, pp. 427-458, 2001. [Online]. Available: http: //stacks.iop.org/0026-1394/38/427

[3] W. M. Itano, L. L. Lewis, and D. J. Wineland, "Shift of ${ }^{2} S_{1 / 2}$ hyperfine splittings due to blackbody radiation," Phys. Rev. A vol. 25, no. 2, pp. 1233-1235, Feb. 1982. [Online]. Available: http://prola.aps.org/abstract/PRA/v25/i2/p1233_1

[4] A. Godone, D. Calonico, F. Levi, S. Micalizio, and C. Calosso, "Starkshift measurement of the ${ }^{2} \mathrm{~S}_{1 / 2}, \mathrm{~F}=3 \longrightarrow \mathrm{F}=4$ hyperfine transition of ${ }^{133}$ Cs," Phys. Rev. A, vol. 71, no. 6, p. 063401, 2005. [Online]. Available: http://link.aps.org/abstract/PRA/v71/e063401
[5] F. Levi, D. Calonico, L. Lorini, S. Micalizio, and A. Godone "Measurement of the blackbody radiation shift of the ${ }^{133} \mathrm{Cs}$ hyperfine transition in an atomic fountain," Phys. Rev. A, vol. 70, no. 3, p. 033412 , 2004. [Online]. Available: http://link.aps.org/abstract/PRA/v70/e033412

[6] E. Simon, P. Laurent, and A. Clairon, "Measurement of the Stark shift of the Cs hyperfine splitting in an atomic fountain," Phys. Rev. A vol. 57, no. 1 , pp. 436-439, Jan. 1998. [Online]. Available: http://prola.aps.org/abstract/PRA/v57/i1/p436_1

[7] A. Bauch and R. Schröder, "Experimental Verification of the Shift of the Cesium Hyperfine Transition Frequency due to Blackbody Radiation," Phys. Rev. Lett., vol. 78, no. 4, pp. 622-625, Jan. 1997. [Online]. Available: http://prola.aps.org/abstract/PRL/v78/i4/p622_1

[8] J. E. Thomas, P. R. Hemmer, S. Ezekiel, C. C. Leiby, R. H Picard, and C. R. Willis, "Observation of Ramsey Fringes Using Stimulated, Resonance Raman Transition in a Sodium Atomic Beam,' Phys. Rev. Lett., vol. 48, no. 13, pp. 867-870, Mar. 1982. [Online] Available: http://prola.aps.org/abstract/PRL/v48/i13/p867_1

[9] J. Fuchs, G. J. Duffy, W. J. Rowlands, and A. M. Akulshin, "Electromagnetically induced transparency in ${ }^{6} \mathrm{Li}, "$ J. Phys. B, vol. 39, no. 17, pp. 3479-3489, 2006. [Online]. Available: http: //stacks.iop.org/0953-4075/39/3479

[10] U. Rasbach, "Magneto-optische Effekte und Messung der elektrischen Tensorpolarisierbarkeit an einem Cäsium-Atomstrahl," Ph.D. dissertation, University of Fribourg, 2001, unpublished.

[11] C. Ospelkaus, U. Rasbach, and A. Weis, "Measurement of the forbidden tensor polarizability of Cs using an all-optical Ramsey resonance technique," Phys. Rev. A, vol. 67, no. 1, p. 011402, Jan. 2003. [Online]. Available: http://prola.aps.org/abstract/PRA/v67/i1/e011402

[12] N. F. Ramsey, Molecular Beams. London: Oxford University Press, 1956.

[13] S. Ulzega, "Theoretical and experimental study of the Stark effect in the ground state of alkali atoms in helium crystals," Ph.D. dissertation, University of Fribourg, Switzerland, 2006

[14] S. Ulzega, A. Hofer, P. Moroshkin, and A. Weis, "Reconciliation of experimental and theoretical electric tensor polarizabilities of the cesium ground state," Europhys. Lett., vol. 76, no. 6, pp. 1074-1080, Dec. 2006, see errata in [17]. [Online]. Available: http://dx.doi.org/10.1209/epl/i2006-10383-2

[15] R. D. Swenumson and U. Even, "Continuous flow reflux oven as the source of an effusive molecular Cs beam," Rev. Sci. Instrum., vol. 52 , pp. 559-561, 1981

[16] F. Levi, A. Godone, J. Vanier, S. Micalizio, and G. Modugno, "Line-shape of dark line and maser emission profile in CPT," Eur. Phys. J. D, vol. 12, pp. 53-59, 2000. [Online]. Available: http://dx.doi.org/10.1007/s100530070042

[17] S. Ulzega, A. Hofer, P. Moroshkin, and A. Weis, "Reconciliation of experimental and theoretical electric tensor polarizabilities of the cesium ground state," Europhys. Lett., vol. 78, no. 6, p. 69901, June 2007. [Online]. Available: http://dx.doi.org/10.1209/0295-5075/78/69901 